\title{
Searches for additional Higgs bosons at the LHC
}

\author{
Junquan Tao*t+ \\ Institute of High Energy Physics, Chinese Academy of Sciences, Beijing 100049, P. R. China \\ E-mail: taojq@ihep.ac.cn
}

\begin{abstract}
Some latest results of the direct searches for additional Higgs bosons at ATLAS and CMS are presented. The searches for heavy neutral Higgs bosons, light neutral Higgs bosons, singly and doubly charged Higgs bosons are performed using proton-proton collision data at $\sqrt{s}=13 \mathrm{TeV}$ collected by the ATLAS and CMS detectors. No significant sign of any beyond standard model Higgs boson is observed. For each search the upper limit on the Higgs boson production cross section times branching ratio is set.
\end{abstract}

The Ninth Annual Conference on Large Hadron Collider Physics - LHCP2021

7-12 June 2021

Online

* Speaker.

† on behalf of the ATLAS and CMS Collaborations

¥Supported by National Natural Science Foundation of China (No. 11661141007, No. 11875275 and No. 12061141003), National Key Research and Development Program of China (No. 2018YFA0403900) and partially by CAS Center for Excellence in Particle Physics (CCEPP). 


\section{Introduction}

In 2012, both the ATLAS [1] and CMS [2, 3] Collaborations observed a new boson with a mass of approximately $125 \mathrm{GeV}$ whose properties are at present compatible with those of the Higgs boson in the standard model (SM) of particle physics. However, many important questions dealing with the nature and origin of the Higgs boson observed at the LHC remain unanswered. Physics beyond the SM (BSM) can also provide a Higgs boson that is compatible with the observed $125 \mathrm{GeV}$ boson. The extended parameter space of several BSM models, such as generalized models containing two Higgs doublets (2HDM) [4] and the next-to-minimal supersymmetric model (NMSSM) [5, 6], give rise to a rich and interesting phenomenology, including the presence of additional Higgs bosons. Searching for additional Higgs bosons is therefore an important way to probe BSM physics and address the naturalness problem in the SM. This paper summarizes some selected results of the latest searches for additional Higgs Bosons in a wide mass range at both ATLAS and CMS. The data set is the proton-proton collisions collected with the ATLAS and CMS detectors at the LHC [7, 8], at a center-of-mass energy of $13 \mathrm{TeV}$.

\section{Heavy neutral Higgs boson searches}

Searches for a new hypothetical scalar particle $(X)$ decaying into SM Higgs boson pairs, or the SM Higgs boson and another scalar particle, are performed by ATLAS and CMS.

A search for $X \rightarrow H H \rightarrow b \bar{b} \gamma \gamma$ is performed by ATLAS with a data set corresponding to an integrated luminosity of $139 \mathrm{fb}^{-1}$ of proton-proton collisions at a center-of-mass energy of $13 \mathrm{TeV}$ [9]. Using the TMVA toolkit [10], two boosted decision trees (BDTs) are trained to better separate the signal from the $\gamma \gamma$ plus the $t \bar{t} \gamma \gamma$ backgrounds and the single Higgs boson background, where $Z H$ and $t \bar{t} H$ production modes are the dominant resonant backgrounds. The combined BDT score of an event, obtained by combining the two BDT scores in quadrature, is used to select events. The signal and backgrounds are extracted by fitting analytic functions to the di-photon invariant mass distribution in the range $105 \mathrm{GeV} \leq m_{\gamma \gamma} \leq 160 \mathrm{GeV}$. No excess with respect to background expectations is found. The observed (expected) limits on the cross section $p p \rightarrow X \rightarrow H H$ range from $610 \mathrm{fb}$ to $47 \mathrm{fb}(360-43 \mathrm{fb})$ over the considered mass range, $251 \mathrm{GeV} \leq m_{X} \leq 1000 \mathrm{GeV}$, as shown in the left plot of Figure 1. The expected limit on the cross section improves by a factor of two to three depending on the $m_{X}$ value, compared to the previous ATLAS result based on $36 \mathrm{fb}^{-1}$ of $13 \mathrm{TeV}$ collisions.

CMS performed the first such search with a heavy neutral Higgs boson $H$ decaying into an SM Higgs boson $h$ and another neutral Higgs boson $h_{S}$ with a mass of $m_{h_{S}}<m_{H}-m_{h}$, using $137 \mathrm{fb}^{-1}$ of proton-proton collisions at a center-of-mass energy of $13 \mathrm{TeV}$ [11]. A promising signature for the search is thus given by the decay of the $h$ into a pair of tau leptons and the decay of the $h_{S}$ into a pair of b quarks, $h\left(\tau_{h} \tau_{h}\right) h_{S}(b \bar{b})$. For the $\tau$ pair, the $e \tau_{h}, \mu \tau_{h}$ and $\tau_{h} \tau_{h}$ final states are used. A mass range of $240-3000 \mathrm{GeV}$ for $m_{H}$ and $60-2800 \mathrm{GeV}$ for $m_{h_{S}}$ is covered. A neural network (NN) multi-classification is used for event categorization and also extracting the signal. No signal has been observed. Model independent 95\% confidence level upper limits on the product of the production cross section and the branching fractions of the signal process are set ranging from $125 \mathrm{fb}$ (for $m_{H}=240 \mathrm{GeV}$ ) to $2.7 \mathrm{fb}$ (for $m_{H}=3000 \mathrm{GeV}$ ), as shown in the middle plot of Figure 1 . 
These limits have been compared to maximally allowed products of the production cross section and the branching fractions of the signal process in the next-to-minimal supersymmetric extension of the standard model, as shown in the right plot of Figure 1.

ATLAS performed the search for a heavy neutral Higgs boson, $A$, decaying into a $\mathrm{Z}$ boson and another heavy Higgs boson, $H$, using a data sample corresponding to an integrated luminosity of $139 \mathrm{fb}^{-1}$ [12]. Two final states were considered, where the $H$ boson decays into a pair of $b$ quarks or $W$ bosons, and in both cases the $Z$ boson decays into a pair of electrons or muons. The analysis requires exactly two oppositely charged muons or electrons. In order to improve the mass resolution of the heavy Higgs boson $A$, the mass of two b-jets from $H$ or 4 jets from $H$ to $W W$ is constrained to agree with the Higgs mass. For the $\ell \ell b b$ final states, 3 event categories according to production modes and the number of $b$-jets, are employed. The data are in good agreement with the background predicted by SM. For $\ell \ell b b$, upper limits are set in the range 6.2-380 fb for gluongluon fusion and 6.8-210 $\mathrm{fb}$ for $b$-associated production of a narrow $A$ boson in the mass range 230-800 GeV, assuming the $H$ boson is in the mass range $130-700 \mathrm{GeV}$. For $\ell \ell W W$, the observed upper limits are in the range 0.023-8.9 pb for gluon-gluon fusion production of a narrow $A$ boson in the mass range $300-800 \mathrm{GeV}$, assuming the $H$ boson is in the mass range $200-700 \mathrm{GeV}$.
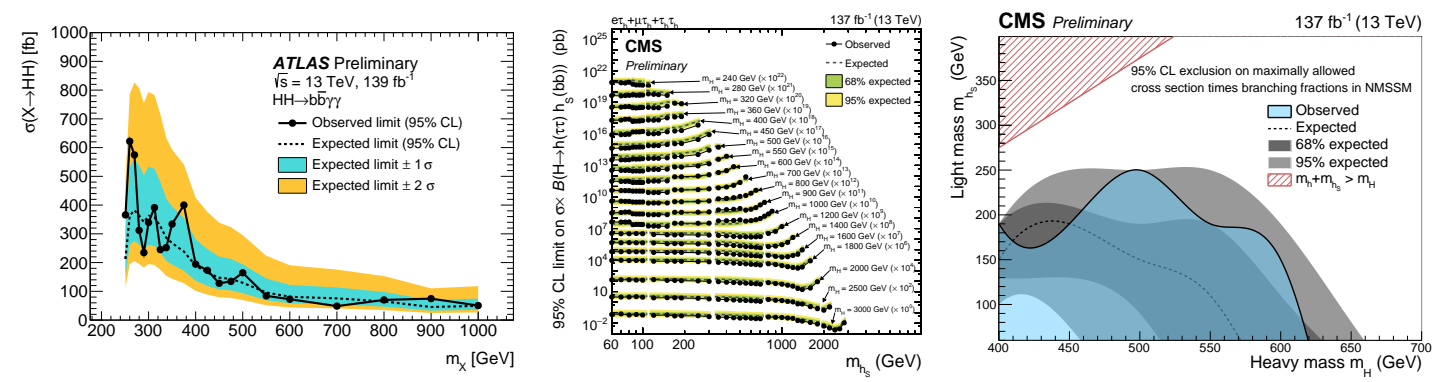

Figure 1: (Left) Observed and expected limits at 95\% confidence level (CL) on the production cross section of a narrow width scalar resonance $X$ as a function of the mass $m_{X}$ of the hypothetical scalar particle, performed by ATLAS with $X \rightarrow H H \rightarrow b \bar{b} \gamma \gamma$ [9]. (Middle) Expected and observed 95\% CL upper limits on $\sigma \times B\left(H \rightarrow h\left(\tau_{h} \tau_{h}\right) h_{S}(b \bar{b})\right)$ for all tested values of $m_{H}$ and $m_{h_{S}}$ [11]. The limits for each corresponding mass value have been scaled by orders of ten as indicated in the annotations. (Right) Mass range in $\mathrm{mH}$ and $\mathrm{mhS}$ for which the maximally allowed $\sigma \times B\left(H \rightarrow h\left(\tau_{h} \tau_{h}\right) h_{S}(b \bar{b})\right)$ within the NMSSM can be excluded at $95 \%$ CL by this CMS search [11].

\section{Light neutral Higgs boson searches}

Searches for light pseudoscalar $a_{1}$ bosons, produced from decays of the $125 \mathrm{GeV}$ Higgs boson $(H)$, in different decay channels are performed at the LHC using the 2016 data set $[13,14,15$, $16,17,18,19,20,21]$. Based on $139 \mathrm{fb}^{-1}$ of $13 \mathrm{TeV}$ collisions, ATLAS performed the search for decays of the observed Higgs boson to a pair of light neutral Higgs bosons, with one $a$-boson decays to a $b$-quark pair and the other to a muon pair [22]. After some basic selections on the dimuon pair, two $b$-tagged jets and missing transverse energy $\left(E_{T}\right)$, a BDT is employed to reduce the SM mainly $t \bar{t}$ and DY backgrounds. A kinematic likelihood fitis performed to improve the 
$\mu \mu b b$ mass resolution, varying the $b$-jet energies within their resolution. The left plot in Figure 2 shows the upper limits on $B(H \rightarrow a a \rightarrow b b \mu \mu)$ as a function of the signal mass $m_{a}$. The upper limits on the branching fraction range between 0.22 to $4 \times 10^{-4}$ depending on the $a$ mass. The limit is improved by a factor of 2-5 over the full $m_{\mu \mu}$ range compared to the previous result with $36 \mathrm{fb}^{-1}$ data, with a factor of $\sim 2$ from the larger data set and a further factor $\sim 2$ from the BDT for the discrimination of the signal against the SM backgrounds. The largest excess is observed at $m_{\mu \mu}=52 \mathrm{GeV}$ with a local significance of $3.3 \sigma$ and a global significance of $1.7 \sigma$.
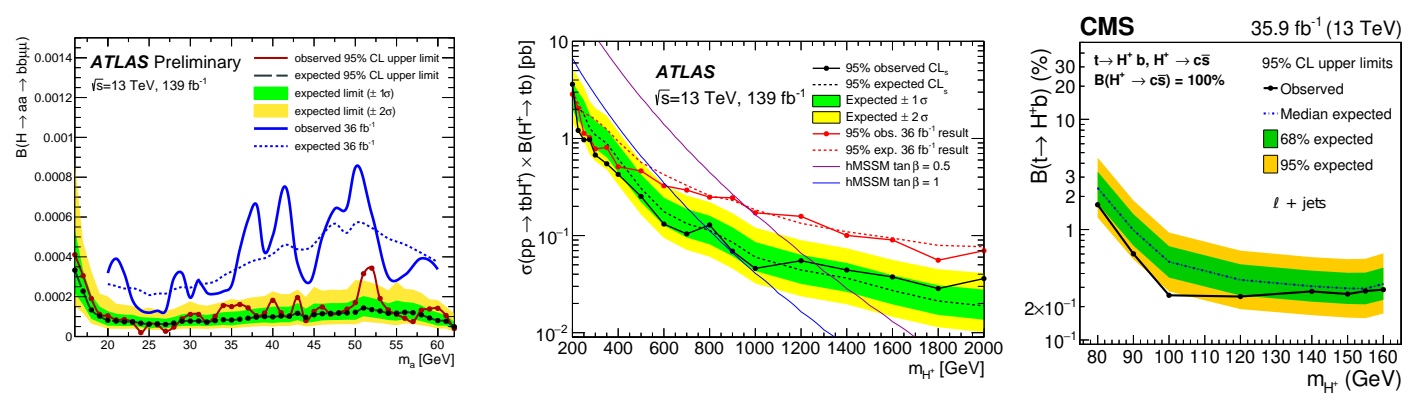

Figure 2: (Left) Upper limits on $B(H \rightarrow a a \rightarrow b b \mu \mu)$ as a function of the signal mass [22]. (Middle) Observed and expected upper limits for the production of $H^{+} \rightarrow t b$ in association with a top quark and a bottom quark [23]. (Right) The expected and observed upper limit in $\%$ on $B\left(t \rightarrow H^{+} b\right)$ as a function of $m_{H^{+}}$using $m_{j j}$ after the individual charm tagging categories have been combined, for the combination of muon+jets and electron+jets channels [24].

\section{Charged Higgs boson searches}

For singly charged Higgs boson decaying into fermions, ATLAS performed the search for charged Higgs decaying into a top quark and a bottom quark using $139 \mathrm{fb}^{-1}$ data [23]. In this search, 1 lepton plus jets events are categorized according to numbers of jets and $b$-jets. A Neural Network is used to discriminate between signal and background events. The main background is $t \bar{t}$ which was estimated from MC but corrected in data control regions. As shown in the middle plot of Figure 2 the observed upper limits on the cross section times the branching ratio range from $3.6 \mathrm{pb}$ at $200 \mathrm{GeV}$ to $36 \mathrm{fb}$ at $2000 \mathrm{GeV}$ at $95 \%$ confidence level, which improved by $5 \%$ to $70 \%$, depending on the $H^{+}$mass, compared to the previous ATLAS search with $36 \mathrm{fb}^{-1}$.

CMS performed the search of singly charged Higgs bosons produced in the process of top quark pair production, where one top quark decays to a bottom quark and a charged Higgs boson and the other to a bottom quark and a $W$ boson, using $35.9 \mathrm{fb}^{-1}$ data set [24]. With the $\mathrm{W}$ boson decaying to a charged lepton (electron or muon) and a neutrino, the final state comprises an isolated lepton, missing transverse momentum, and at least four jets, of which two are tagged as $b$ jets. To enhance the search sensitivity, one of the jets originating from the charged Higgs boson is required to satisfy a charm tagging selection. An upper limit in the range $1.68 \%-0.25 \%$ is set on the branching fraction of the top quark decay to the charged Higgs boson and bottom quark for a charged Higgs boson mass between 80 and $160 \mathrm{GeV}$, as shown in the right plot of Figure 2. 
ATLAS performed the doubly charged Higgs search in the pair production of doubly charged $H^{ \pm \pm}$bosons, or the associated production of a doubly charged $H^{ \pm \pm}$boson and a singly charged $H^{ \pm}$boson using $139 \mathrm{fb}^{-1}$ data [25]. The search is guided by a type-II seesaw model that extends the scalar sector of SM with a scalar triplet [26]. Three final states including two same-charge leptons, three leptons, and four leptons, are considered. Combining those channels, $H^{ \pm \pm}$bosons are excluded at $95 \%$ confidence level up to $350 \mathrm{GeV}$ and $230 \mathrm{GeV}$ for the pair and associated production modes, respectively.

CMS investigated the singly and doubly charged Higgs bosons in a context of Georgi-Machacek (GM) model [27] using $137 \mathrm{fb}^{-1}$ data [28]. Both charged Higgs boson are produced in vector boson fusion processes and decaying into vector bosons. The search was performed in fully leptonic decays of the vector bosons. Events are selected by requiring two or three electrons or muons, moderate missing transverse momentum, and two jets with a large rapidity separation and a large dijet mass. The exclusion limits on the product of the charged Higgs boson cross section and branching fraction, as a function of Higgs mass from $200 \mathrm{GeV}$ to $3 \mathrm{TeV}$, are shown in Figure 3 with the left plot for doubly charged Higgs and the middle plot for singly charged Higgs. The observed 95\% confidence level limits exclude GM $s_{H}$ parameter values greater than $0.20-0.35$ for the mass range from 200 to $1500 \mathrm{GeV}$, as shown in the right plot of Figure 3 .
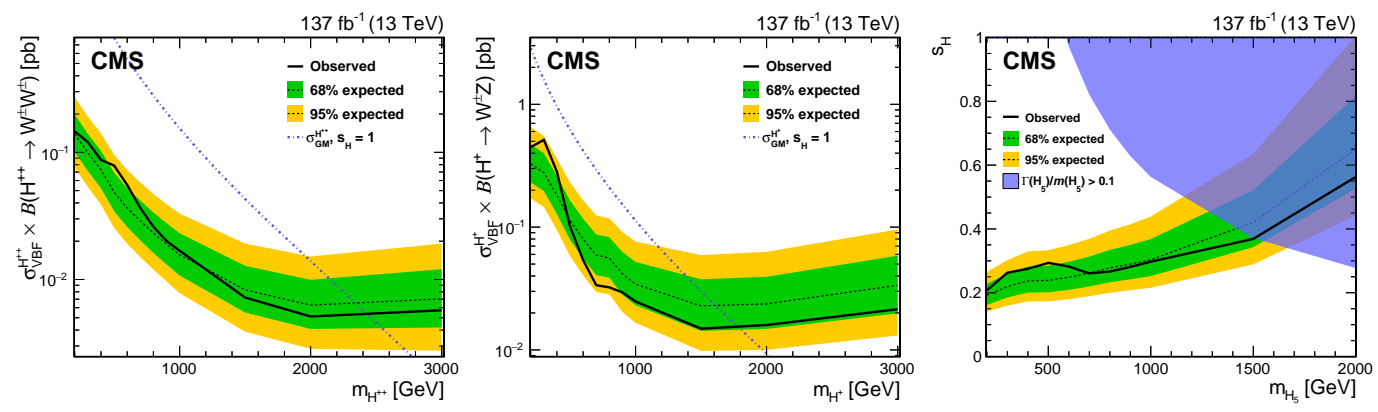

Figure 3: Expected and observed exclusion limits at $95 \% \mathrm{CL}$ for $\sigma_{\mathrm{VBF}}\left(H^{ \pm \pm}\right) B\left(H^{ \pm \pm} \rightarrow W W\right)$ as functions of $m_{H^{ \pm \pm}}$(left), for $\sigma_{\mathrm{VBF}}\left(H^{ \pm}\right) B\left(H^{ \pm} \rightarrow W Z\right.$ ) as functions of $m_{H^{ \pm}}$(middle), and for $s_{H}$ as functions of $m_{H_{5}}$ in the GM model (right) [28]. The contribution of the $H^{ \pm}\left(H^{ \pm \pm}\right)$boson signal is set to zero for the derivation of the exclusion limits on the $\sigma_{\mathrm{VBF}}\left(H^{ \pm \pm}\right) B\left(H^{ \pm \pm} \rightarrow W W\right)\left(\sigma_{\mathrm{VBF}}\left(H^{ \pm}\right) B\left(H^{ \pm} \rightarrow W Z\right)\right)$. The exclusion limits for $s_{H}$ are shown up to $m_{H_{5}}=2000 \mathrm{GeV}$, given the low sensitivity in the GM model for values above that mass. Values above the curves are excluded.

\section{Summary}

Some latest results of the searches for additional Higgs bosons, including heavy neutral Higgs bosons, light neutral Higgs bosons, singly and doubly charged Higgs bosons, in a wide mass range at the LHC are presented. No significant sign of any beyond the standard model Higgs boson is observed. The upper limits on the Higgs boson production cross section times branching ratio are set. For some searches, the results are interpreted in the context of some BSM models. 


\section{References}

[1] ATLAS Collaboration, Phys. Lett. B 716 (2012) 1 [arXiv:1207.7214 [hep-ex]].

[2] CMS Collaboration, Phys. Lett. B 716 (2012) 30 [arXiv:1207.7235 [hep-ex]].

[3] CMS Collaboration, JHEP 1306 (2013) 081 [arXiv:1303.4571 [hep-ex]].

[4] G. Cacciapaglia, A. Deandrea, S. Gascon-Shotkin, S. Le Corre, M. Lethuillier and J. Tao, JHEP 1612 (2016) 068 [arXiv:1607.08653 [hep-ph]].

[5] J. W. Fan et al., Chin. Phys. C 38 (2014) 073101 [arXiv:1309.6394 [hep-ph]].

[6] J. Q. Tao et al., Chin. Phys. C 42 (2018) 103107 [arXiv:1805.11438 [hep-ph]].

[7] ATLAS Collaboration, JINST 3 (2008), S08003 doi:10.1088/1748-0221/3/08/S08003

[8] CMS Collaboration, JINST 3 (2008), S08004 doi:10.1088/1748-0221/3/08/S08004

[9] ATLAS Collaboration, ATLAS-CONF-2021-016, http://cds.cern.ch/record/2759683.

[10] A. Hocker, P. Speckmayer, J. Stelzer, J. Therhaag, E. von Toerne, H. Voss, M. Backes, T. Carli, O. Cohen and A. Christov, et al. arXiv:physics/0703039 [physics.data-an].

[11] CMS Collaboration, CMS-PAS-HIG-20-014, http://cds.cern.ch/record/2758271.

[12] ATLAS Collaboration, Eur. Phys. J. C 81 (2021) no.5, 396 doi:10.1140/epjc/s10052-021-09117-5 [arXiv:2011.05639 [hep-ex]].

[13] CMS Collaboration, Phys. Lett. B 796 (2019) 131 [arXiv:1812.00380 [hep-ex]].

[14] CMS Collaboration, arXiv:1907.07235 [hep-ex].

[15] CMS Collaboration, Phys. Lett. B 795 (2019) 398 [arXiv:1812.06359 [hep-ex]].

[16] CMS Collaboration, JHEP 1811 (2018) 018 [arXiv:1805.04865 [hep-ex]].

[17] CMS Collaboration, Phys. Lett. B 785 (2018) 462 [arXiv:1805.10191 [hep-ex]].

[18] ATLAS Collaboration, JHEP 06 (2018), 166 doi:10.1007/JHEP06(2018)166 [arXiv:1802.03388 [hep-ex]].

[19] ATLAS Collaboration, JHEP 10 (2018), 031 doi:10.1007/JHEP10(2018)031 [arXiv:1806.07355 [hep-ex]].

[20] ATLAS Collaboration, Phys. Rev. D 102 (2020) no.11, 112006 doi:10.1103/PhysRevD.102.112006 [arXiv:2005.12236 [hep-ex]].

[21] ATLAS Collaboration, Phys. Lett. B 782 (2018), 750-767 doi:10.1016/j.physletb.2018.06.011 [arXiv:1803.11145 [hep-ex]].

[22] ATLAS Collaboration, ATLAS-CONF-2021-009, http://cds.cern.ch/record/2759283.

[23] ATLAS Collaboration, JHEP 06 (2021), 145 doi:10.1007/JHEP06(2021)145 [arXiv:2102.10076 [hep-ex]].

[24] CMS Collaboration, Phys. Rev. D 102 (2020) no.7, 072001 doi:10.1103/PhysRevD.102.072001 [arXiv:2005.08900 [hep-ex]].

[25] ATLAS Collaboration, JHEP 06 (2021), 146 doi:10.1007/JHEP06(2021)146 [arXiv:2101.11961 [hep-ex]].

[26] J. Schechter and J. W. F. Valle, Phys. Rev. D 22 (1980), 2227 doi:10.1103/PhysRevD.22.2227 
[27] H. Georgi and M. Machacek, Nucl. Phys. B 262 (1985), 463-477 doi:10.1016/0550-3213(85)90325-6

[28] CMS Collaboration, Eur. Phys. J. C 81 (2021) no.8, 723 doi:10.1140/epjc/s10052-021-09472-3 [arXiv:2104.04762 [hep-ex]]. 